\title{
Síndrome del opérculo torácico superior unilateral en un caso con costillas cervicales bilaterales
}

\section{Unilateral thoracic outlet syndrome in a case with bilateral cervical ribs}

\author{
Juan Miguel Alemán-Iñiguez, ${ }^{*}$ Hernán Hermida-Córdova ${ }^{\ddagger}$
}

*Universidad San Francisco de Quito, Ecuador; ${ }^{*}$ Universidad de Cuenca, Ecuador.

\begin{abstract}
RESUMEN. La costilla cervical puede ser un raro hallazgo en sujetos asintomáticos. Cuando produce compresión afecta variablemente las estructuras vasculonerviosas del plexo braquial; el cuadro clínico es progresivo, relacionado al movimiento; el diagnóstico es por exclusión, los estudios de imagen indican el sitio de conflicto osteoneurovascular, $y$ los electrofisiológicos indican el grado de afección neural. A continuación presentamos un paciente joven con historia de ocho meses de paresia y disestesias derechas, con imagen de costillas cervicales bilaterales con diferentes características anatómicas. Con este caso se discute cómo una variante de la anatomía produce patología y cuándo no está relacionado a compresión.
\end{abstract}

Palabras clave: Costilla cervical, plexo braquial, opérculo torácico.

\section{INTRODUCCIÓN}

La costilla cervical (CC), cuya sinonimia es «costilla supernumeraria de Eve» (OMIM:117900), consiste en una costilla accesoria que se articula con las apófisis transversas de C5 o C6. Es una variable anatómica con prevalencia diversa dependiendo de las diferentes poblaciones entre 0.5-7\%, 90\% de los casos son asintomáticos, la prevalencia aumenta en sujetos con tumores del ectodermo, sobre todo de las crestas neurales y mesenquimales (23-33\%) y asociado en $30 \%$ de pacientes con síndrome del opérculo cervicotorácico (SOCT). ${ }^{1,2}$ Los síntomas y signos son vasculonerviosos por compresión a nivel del segmento supraclavicular del plexo braquial (PB), con afección sobre todo al tronco inferior, en otras menos frecuentes,

\section{Correspondencia:}

\section{Dr. Juan Miguel Alemán-Iñiguez}

Universidad San Francisco de Quito, Ecuador.

Correo electrónico: juanmig_18@hotmail.com

Recibido: 29-XII-2020; aceptado: 05-II-2021.

Citar como: Alemán-Iñiguez JM, Hermida-Córdova H. Síndrome del opérculo torácico superior unilateral en un caso con costillas cervicales bilaterales. Neumol Cir Torax. 2021; 80 (2): 122-127. https://dx.doi.org/10.35366/100994
ABSTRACT. The cervical rib can be a rare finding in asymptomatic subjects. When it produces compression, it affects variably the vasculonervous structures of the brachial plexus; the clinical is progressive, related to movement; diagnosis is by exclusion, imaging studies indicate the site of osteo-neuro-vascular conflict and electrophysiological studies indicate the degree of neural involvement. Next, we present a young male patient with an eight-month history of paresis and right dysesthesias, with an image of bilateral cervical ribs with different anatomical characteristics; this case discusses how a variant of the anatomy produces pathology and when it is not related to compression.

Keywords: Rib cervical, brachial plexus, thoracic outlet.

infraclavicular a los fascículos posterior y medial del PB; el lugar del conflicto neurovascular óseo (CONV) se encuentra en el pasadizo donde el plexo cambia su orientación horizontal a vertical; ${ }^{3-5}$ su patogenia tiene bases embriológicas, y con fenotipos en poblaciones con pleuroapófisis vestigiales, «recuerdos evolutivos». En otros casos se ha relacionado a las mutaciones en los genes Hox y la desactivación del factor de diferenciación del crecimiento 11 en la cuarta semana de embriogénesis resultando en falla en la diferenciación de los esclerotomas, por lo que se le considera anomalía congénita; ${ }^{6}$ afecta mayoritariamente a mujeres $(2: 1)$.

La sintomatología más común es la cervicobraquialgia, acompañada de parestesias y disminución de fuerza de los músculos de la región tenar, compromiso de los territorios de los nervios ulnar y mediano, la evolución clínica es lenta. Al ser una patología ósea el diagnóstico se fundamenta en la reconstrucción tomográfica, los estudios electrofisiológicos y neurografías por resonancia son para constatar el grado de afección del PB y su potencial de recuperación. ${ }^{3,5} \mathrm{~A}$ continuación se discuten las características, asociaciones y condiciones para que una variable de la normalidad produzca enfermedad en el contexto de un paciente con costillas cervicales bilaterales donde únicamente existe patología unilateral. 
Neumol Cir Torax. 2021; 80 (2): 122-127

\section{PRESENTACIÓN DEL CASO}

Paciente masculino de 27 años que acude a consulta del Hospital José Carrasco Arteaga por cuadro de ocho meses de evolución de sensación de parestesias y disestesia (HP:0003401 y HP:0012534) en dermatomas C7 y C8 del lado derecho, presencia de prominentes pliegues tenares y palmares proximales («mano simiana» HP:0000954), disminución de la fuerza en el grupo de los músculos lumbricales (HP:0002460), en la flexión de los dedos y signo de Froment positiva, prueba de provocación de Roos positiva con caída de miembro superior derecho; signos de claudicación vascular y prueba de Adson y Wright positivos con disminución del pulso radial derecho.

El estudio de electroconducción reveló disminución del reclutamiento de las fibras: $1^{\circ}$ interóseo dorsal derecho, extensor del índice derecho, con normalidad de los grupos proximales del miembro superior al igual que el de los flexores y extensores comunes de los dedos, hallazgos de afección tronco inferior del PB derecho, el lado izquierdo no tuvo síntomas ni signos en el examen físico, la electroconducción indicó normalidad en el lado izquierdo. En imagen, la radiografía de tórax y tomografía demostraron costillas cervicales: derecha con el extremo distal libre y la izquierda con su extremo distal articulada en la cara posterior del tubérculo del músculo escaleno anterior de la primera costilla. En el estudio vascular de reconstrucción venosa se observó diminución calibre de la vena axilar derecha en el lugar del CONV (Figuras $1 A-C$ y $2 A$ ).

Se realizó intervención quirúrgica, exéresis de CC y abordaje supraclavicular, se evidenció CC derecha articulada a la apófisis transversa C6, con borde libre estrechando el opérculo torácico (OCT), el tronco nervioso inferior del PB derecho se encontró comprimido y deformado, se realizó neurólisis, adherenciólisis del lugar del conflicto con trasposición, liberación del segmento comprimido y fresado del borde superior de la primera costilla (Figura 2B).

El paciente en su evolución tuvo mejoría sintomática a los tres meses y con rehabilitación física mejoró la fuerza de aprehensión palmar; sin embargo, persistieron los valores de reclutamientos bajos en los grupos musculares distales, aun sin sintomatología ni alteración en la electroconducción en el lado izquierdo.

\section{DISCUSIÓN}

La segunda forma más frecuente de CC es la bilateral, la CC unilateral derecha es la más presente. De acuerdo al tamaño y al desarrollo las costillas cervicales pueden ser: I: completa con su borde distal articulado, II: incompleta con su extremo distal libre, III: incompleta con banda fibrosa que la une al tórax y IV: como banda ósea corta que se puede confundir con una apofisomegalia transversa; ${ }^{3-7}$ las características anatómicas que pueden aumentar la posibilidad de compresión neurovascular aún no se han esclarecido por completo. Sin embargo, la presencia de borde libre verdadero, que ante el movimiento oblitera las estructuras del OCT es una teoría fuertemente sustentada, cuando en la imagen se distingue borde libre y el paciente
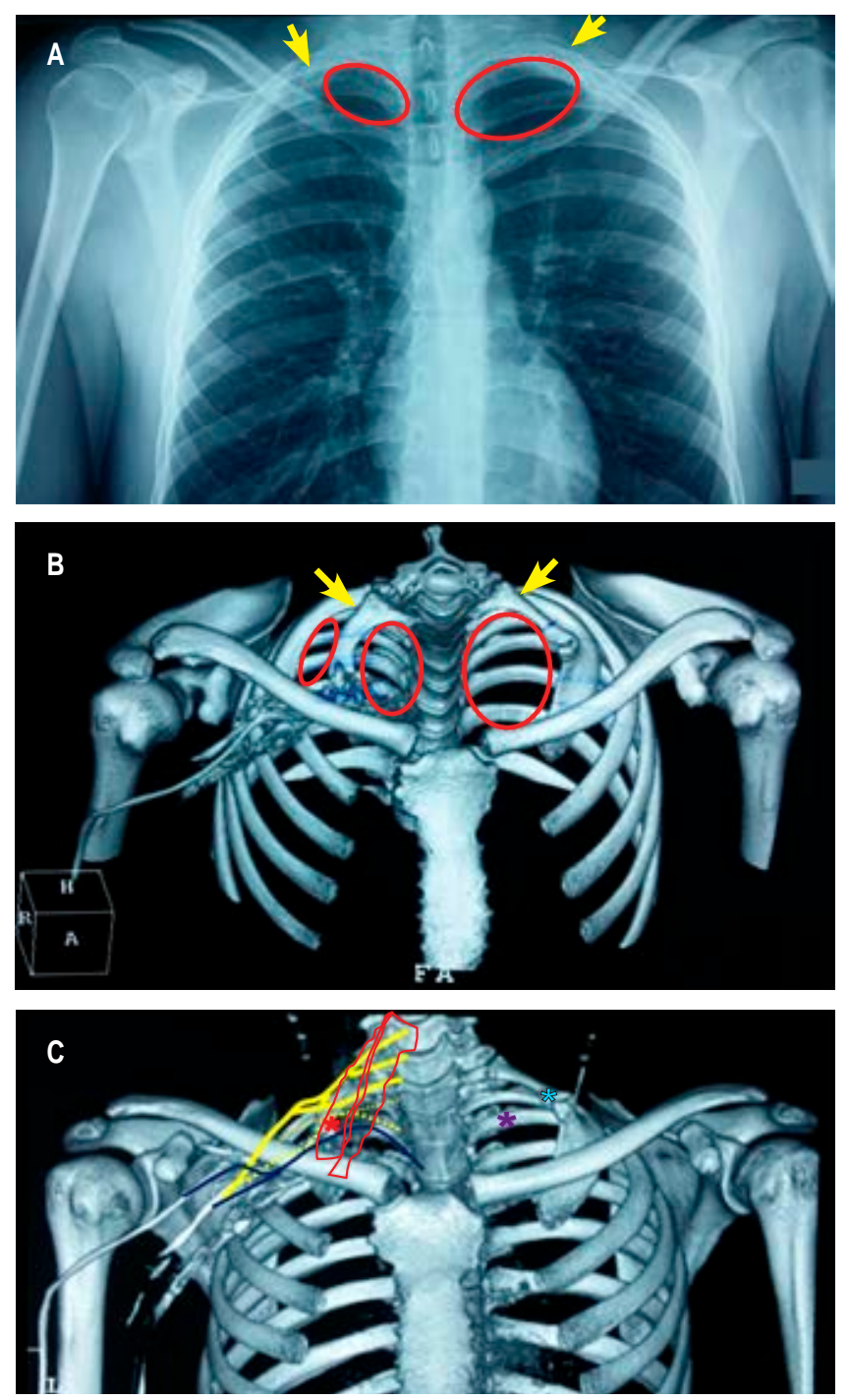

Figura 1: A) Imagen de radiografía estándar de tórax, la apertura torácica superior derecha disminuida en comparación con la izquierda (líneas rojas) y costillas accesorias bilaterales (flechas amarillas). B) Reconstrucción tomográfica tridimensional ósea y angiotomografía venosa derecha. Comparación de los opérculos torácicos (líneas rojas), el derecho dividido en dos y el izquierdo sin división, costillas accesorias (flechas amarillas). C) Plexo braquial derecho (líneas amarillas), tronco inferior del plexo braquial derecho (línea interpunteada amarilla), músculos escaleno anterior y posterior (línea roja), vena axilar (línea azul), conflicto osteoneurovascular derecho (asterisco rojo), opérculo cervicotorácico izquierdo libre (asterisco púrpura) y borde distal izquierdo articulado a la primera costilla (asterisco cardenillo). 

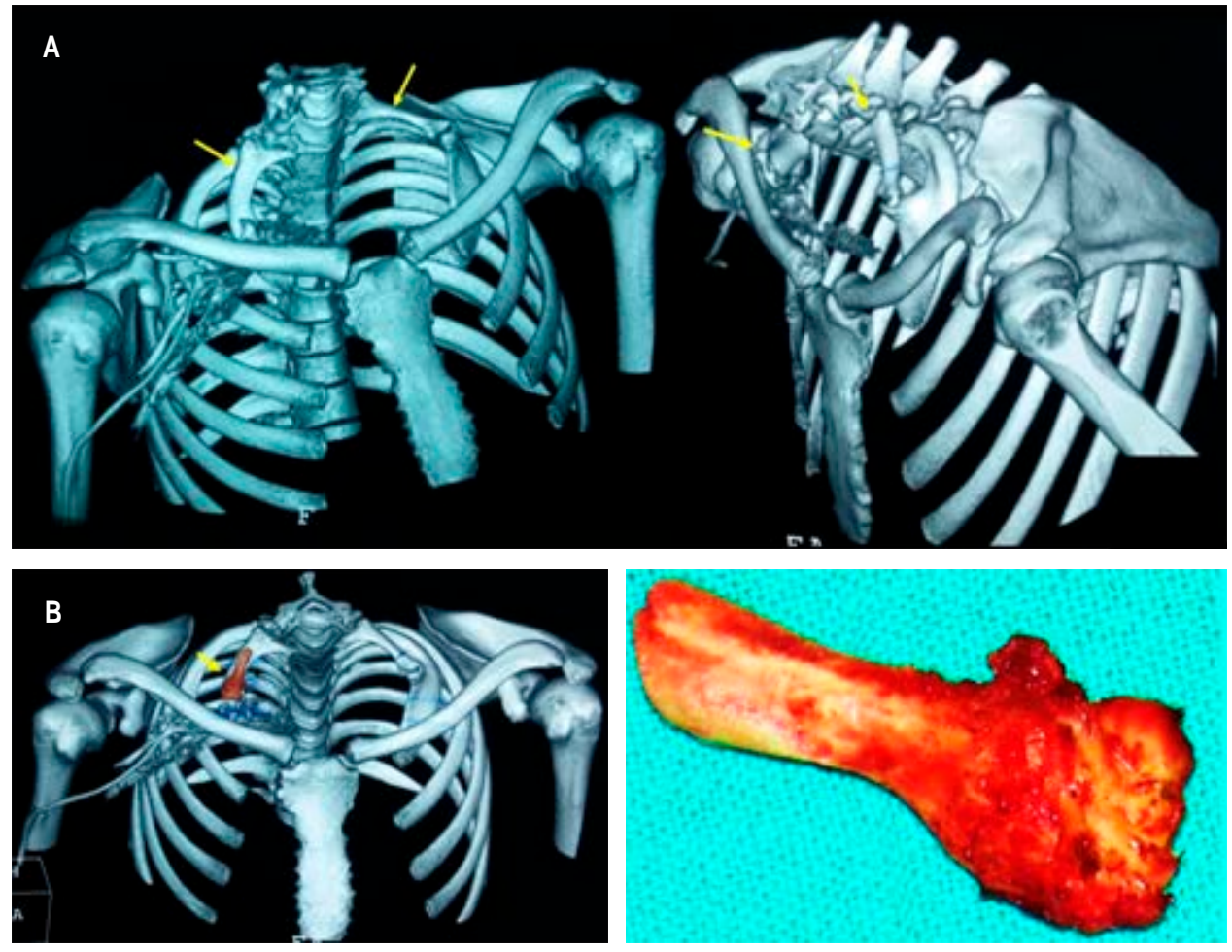

Figura 2:

A) Reconstrucción tomográfica tridimensional ósea y visiones? perfiladas de las costillas accesorias (flechas amarillas). B) Espécimen del extremo distal de la costilla accesoria que fue resecada en dos partes y su ubicación en la tomografía en reconstrucción ósea tridimensional.

es asintomático, la posibilidad más explicable es que se trate de una articulación fibrosa en el extremo libre que estabilice la costilla accesoria, ya que este tejido fibroso no se distingue en las imágenes de reconstrucción ósea. ${ }^{8}$ La sintomatología más frecuente y más consultada por el paciente es el SOCT neural, aunque es una condición sine qua non que las estructuras vasculares estén comprometidas, ${ }^{9}$ un punto importante en el diagnóstico de exclusión es la presencia de signos de claudicación vascular o llamadas pruebas de provocación, pues tienen mayor sensibilidad (pruebas de Adson, Falconer y Weddell y Wright), que lo diferencian de otras plexopatías. ${ }^{9}$

En el caso que presentamos, el paciente consulta por cuadro de cervicobraquialgia derecha con evidencia de neuropatía del tronco inferior del PB, la imagen demostró el lado derecho la costilla accesoria con borde libre, lugar del CONV (tipo II), mientras que el lado izquierdo sin sintomatología, la costilla accesoria tiene articulación verdadera con la primera costilla (Tipo I), el examen físico demostró con las pruebas de provocación compromiso vascular, por lo que revela un dato importante de SOCT arterial y neural.

La historia natural es de evolución lenta, y se requiere estrés repetitivo para presentar sintomatología, por ser una patología congénita el tiempo de diagnóstico es tardío, más frecuente en los adultos de mediana edad, lo que demuestra el fenómeno de estrés prolongado para reducir y atrapar el compartimento cervicotorácico, ${ }^{2,9}$ el daño nervioso muchas veces es tipo neuropraxia y tras el tratamiento tiene buen pronóstico. Aunque la presencia de datos de atrofia de músculos distales son signos de recuperación más lenta, inclusive incompleta, ${ }^{5}$ los estudios electrofisiológicos sobre todo la velocidad de conducción puede ser herramienta diagnóstica, existen criterios quirúrgicos con evidencia de valores menores de $60 \mathrm{~m} / \mathrm{s} ;{ }^{9}$ la electromiografía sirve para descartar otras causas y puede predecir la posibilidad de recuperación principalmente la latencia de la onda F; $y$ en cuanto a imagen, la neurografía por resonancia magnética del PB puede mostrar amputación de la fibras que correspondería a un compromiso avanzado. ${ }^{5}$ En este caso, el examen físico evidenció signos de atrofia distal, pero no se evidenció deformidad como mano en garra o de predicador, en los hallazgos intraoperatorios no observamos cambios permanentes e irreversibles del plexo. A los tres meses el paciente tuvo mejoría clínica y laboral, aunque los estudios electrofisiológicos aún estaban afectados, demuestran que su uso temprano posquirúrgico no es un buen predictor de evolución ni pronóstico.

Los estadíos avanzados de compresión afectan al componente vascular, mientras que, como se mencionó, la sintomatología y el daño neural son más tempranos, pueden observarse afecciones tardías como pseudoaneurismas y trombosis en estudios vasculares como angiografía y angiotomografía, incluso ecografía endovascular para detección de estenosis; ${ }^{9,10}$ éstas son condiciones que ensombrecen el pronóstico y la recuperación. En nuestro caso hubo concurrencia de claudicación nerviosa y vascular, en el intraoperatorio se encontraron las estructuras indemnes. 
Neumol Cir Torax. 2021; 80 (2): 122-127

Las características anatómicas de vulnerabilidad para el CONV independientes de la presencia de CC mencionadas por la literatura y en estudios de autopsia son: tronco superior del PB en posición anterior al músculo escaleno anterior, el tronco superior o inferior que atraviesan los vientres musculares del escaleno anterior y medio, músculo escaleno mínimo y accesorio que comprime los troncos y cordones del plexo, bandas de tejido ligamentoso o fibrofascial desde la parte posterior desde la primera costilla hasta las inserciones en el tórax, tronco inferior y nervio frénico accesorio que pasa por delante de la vena subclavia antes de girar hacia el pecho, clavícula bífida; todas estas características son difíciles de distinguir en estudios de imagen, ${ }^{11}$ pueden estar coexistiendo con la CC y se evidencian intraoperatoriamente; en cuanto a la asociación entre ellas, no está descrita ni es consistentemente causa de la compresión. Por lo tanto, la adherenciólisis y la transposición de estructuras son maniobras que deben acompañar a los procedimientos de exéresis de costillas accesorias, mismas que fueron practicadas en el caso.

Otros estudios de la anatomía y conformación de la caja torácica y su apertura superior también pueden aumentar la posibilidad de compresión independientemente de la presencia de una costilla accesoria, estas son: espacio costoclavicular estrecho en elevación del miembro superior, escoliosis cervicodorsal, gran grosor del músculo subclavio y la disminución de la distancia entre el borde del músculo pectoral menor y el revestimiento posterior axilar, hipertrofia mamaria. Se han evidenciado diferencias de estas variables en sujetos sintomáticos, estadísticamente significativas versus sujetos asintomáticos, estos estudios tienen limitaciones en describir las posiciones y maniobras usadas para estandarizar la medición, ${ }^{8,11}$ son asociaciones antropométricas que deben ser valoradas en la planificación quirúrgica y pueden ser parte del éxito posquirúrgico. En nuestro caso no se constataron alteraciones antropométricas visibles.

Otros artículos citan el estrés psicosocial y síndromes miofasciales en pacientes con diagnóstico de SOCT y sintomatología persistente posterior a exéresis de la costilla accesoria. En cuanto al dolor, al ser un tipo neuropático, la evolución mayor de 12 meses de dolor prequirúrgico es suficiente para producir fenómenos de centralización del dolor crónico que inciden en que el paciente mantenga dolor posoperatorio. ${ }^{2,8,11}$ En nuestro caso, el paciente acudió con dolor, pero no fue el motivo de consulta, tuvieron mayor relevancia los síntomas motores, el caso no evolucionó con dolor neuropático crónico.

Las variaciones del PB son excepcionales y pueden ser: prefijado (con raíces de C4 a C8/T1), posfijado (con raíces de C5/C6 a T2), con comunicación a C4 y con comunicación a T2. No existe evidencia de una susceptibilidad definitiva a SOCT; no obstante, pueden predisponer a plexopatía en ausencia de costillas cervicales; ${ }^{12}$ cuando se asocia a una costilla accesoria se puede plantear que los plexos braquiales posfijados y con comunicación T2 son los más inferiores y más inclinados y por ende, mayor posibilidad de obturarse con una CC. En nuestro caso no se halló variabilidad en la situación del PB.

La CC tiene naturaleza congénita, su aparición en varones se da en la edad adulta temprana, relacionada a la edad de máxima actividad laboral (militares, trabajos con carga de peso, trabajo de oficina) y física (movimientos repetitivos y deportes tales como natación, básquetbol, voleibol y tenis), otro pico se observa en mujeres de edad adulta mediana con afecciones y pérdida de masa muscular. ${ }^{13} \mathrm{El}$ caso que presentamos obedece al primer pico de edad en hombres.

El SOCT, con pruebas de provocación positivas, afección electroconducción del PB y evidencia en imagen de con-

Tabla 1: Resumen de las variantes y opciones en cirugía de la costilla accesoria con compresión del opérculo torácico superior.

\begin{tabular}{|c|c|c|c|}
\hline Abordajes & Vías & Descomprensión & Maniobras auxiliares \\
\hline
\end{tabular}


Tabla 2: Resumen y comparación de los escenarios y tratamientos de la costilla accesoria.

\begin{tabular}{|l|l|}
\hline \multicolumn{1}{|c|}{ Cuando produce compresión } & \multicolumn{1}{c|}{ Cuando no produce compresión } \\
\hline $\begin{array}{l}\text { - Borde libre distal (tipo II y IV) } \\
\text { - Costilla accesoria angulada } \\
\text { - Costilla accesoria mayor de } 3 \mathrm{~cm} \\
\text { - Compartimento opérculo torácico dividido en dos espacios por la } \\
\text { costilla accesoria }\end{array}$ & $\begin{array}{l}\text { - Borde libre distal articulado con la primera costilla (tipo I y III) } \\
\text { - Costilla accesoria sin angulación } \\
\text { - Costilla accesoria menor de } 3 \mathrm{~cm}\end{array}$ \\
& $\begin{array}{l}\text { - Evidencia de otros factores antropométricos que disminuyen el } \\
\text { opérculo torácico }\end{array}$ \\
& $\begin{array}{l}\text { Evidencia de malformaciones y variabilidades de los músculos } \\
\text { escalenos, del plexo braquial y la caja torácica }\end{array}$ \\
& $\begin{array}{l}\text { Evidencia de masas u otras ocupaciones del compartimento } \\
\text { cervicotorácico (intraoperculares, ejemplo: tumor de Pancoast, } \\
\text { extraoperculares, ejemplo: tumores de mama) }\end{array}$ \\
\hline \multicolumn{1}{|c|}{ Tratamiento } \\
\hline $\begin{array}{l}\text { Abordajes convencionales + descompresión única con/sin maniobras } \\
\text { auxiliares a la descompresión }\end{array}$ & $\begin{array}{l}\text { Observación, tratamiento conservador (analgesia + fisioterapia). } \\
\text { Exéresis de masas y otras ocupaciones + abordajes combinados + } \\
\text { descompresión combinada + maniobras auxiliares a la descompresión }\end{array}$ \\
\hline
\end{tabular}

diciones de CC con características a favor de compresión (estudios angiográficos y de plexografía por resonancia), tiene como tratamiento principal la descompresión quirúrgica. Este objetivo se puede lograr con el retiro de la primera costilla, el músculo escaleno anterior, la costilla accesoria o combinación entre ellas; la resección de las dos primeras y sus combinaciones aumentan el tiempo operatorio, el riesgo anestésico, los días de hospitalización y el tiempo de recuperación sin haber diferencia en mejores resultados funcionales frente a la resección única de la CC accesoria con apófisis transversa vertebral distal, esta última se ha asociado a mejoría posquirúrgica de la funcionalidad en las pruebas de Roos y de estrés por elevación del brazo y seguridad en cuanto a complicaciones.

Los abordajes mencionados en la literatura son: supraclavicular, que ofrece visualización y control del PB y de la arteria subclavia; el abordaje transaxilar que permite la resección de la primera costilla y la visualización de la arteria axilar; y los abordajes que se usan menos: la subescapular posterior, la transtorácica y las combinadas: supraclavicular con transaxilar y supraclavicular con infraclavicular para casos de recidivas, costillas cervicales grandes y asociaciones a aneurismas. ${ }^{14,15}$ La vía puede ser convencional microquirúrgica y en centros de experiencia por videotoracoscopia que trae mayor dificultad en la luxación de la primera costilla, sobre todo en pacientes con gran musculatura. ${ }^{16}$

Como mencionamos, según los hallazgos intraoperatorios se pueden observar distintas patologías que son concurrentes a la presencia de la primera costilla. Por lo tanto, se destacan procedimientos auxiliares como adherenciólisis y neurólisis del PB que se usan en presencia de bandas fibromusculares y ligamentarias anexas en casos de recidivas; en evidencia de tronco inferior deformado y desplazado son necesarias la liberación y la transposición neural, además se mencionan el fresado y exéresis parcial de la primera costilla. ${ }^{14-18}$ Por último, el compromiso vascular exige complemento de manejos tales como el endovascular y bypass vasculares para resolución de pseudoaneurismas y trombosis. ${ }^{9,17,18}$ En nuestro caso utilizamos el abordaje supraclavicular, realizamos exéresis de la CC en dos partes, la última incluyó la cabeza y parte la apófisis transversa vertebral, visualizamos las estructuras del PB indemnes, pero deformadas, por lo que procedimos a realizar adherenciólisis, liberación, neurólisis y transposición del tronco inferior para aumentar la posibilidad de éxito posoperatorio y evitar las recidivas (Tablas 1 y 2).

\section{CONCLUSIONES}

Aunque la CC es una variable de la normalidad, debe reunir condiciones anatómicas y asociaciones para producir enfermedad, los escenarios posibles son: sujetos asintomáticos que se someten a exámenes de imagen con evidencia de esta variabilidad, otros que tengan plexopatía y el origen de ésta sea la compresión directa por CC y por último, individuos con plexopatía y CC con otros factores que estrechen el OCT e indirectamente produzcan compresión con la costilla accesoria. Es necesaria la individualización de los casos para tomar la decisión de intervención quirúrgica adecuada, además los hallazgos intraoperatorios determinan las decisiones que garanticen el éxito posoperatorio. En la actualidad el manejo de esta patología no tiene un único objetivo de descompresión, sino de prevenir la recidiva y tratar los factores asociados. En este caso el lado de la sintomatología fue producido por la CC derecha con características de compresión en su borde libre; mientras 
que en el lado izquierdo la CC no tiene características de producir patología. Por lo tanto, es un caso que ilustra la coexistencia de una condición que puede tener dualidad: ser una variante anatómica de la normalidad y ser una patología que requiere diagnóstico y tratamiento de descompresión y las maniobras que garanticen el éxito posquirúrgico.

\section{REFERENCIAS}

1. Schumacher R, Mai A, Gutjahr P. Association of rib anomalies and malignancy in childhood. Eur J Pediatr. 1992;151(6):432-434. Available in: https://doi.org/10.1007/bf01959357

2. Urschel HC, Kourlis H. Thoracic outlet syndrome: a 50-year experience at Baylor University Medical Center. Proc (Bayl Univ Med Cent). 2007;20(2):125-135. Available in: https://doi.org/10.1080/0899 8280.2007 .11928267

3. Atasoy E. Thoracic outlet syndrome: anatomy. Hand Clin. 2004;20(1):714. v. Available in: https://doi.org/10.1016/s0749-0712(03)00078-7

4. Abimbola EO, Willido AA. Prevalence of cervical ribs in a Nigeria population. J Dent Med Sci. 2014;13(2):5-7.

5. Henry BM, Vikse J, Sanna B, Taterra D, Gomulska M, Pekala PA, et al. Cervical rib prevalence and its association with thoracic outlet syndrome: a meta-analysis of 141 studies with surgical considerations. World Neurosurg. 2018;110:e965-e978. Available in: https://doi. org/10.1016/j.wneu.2017.11.148

6. Ten Broek CM, Bakker AJ, Varela-Lasheras I, Bugiani M, van Dongen S, Galis F. Evo-devo of the human vertebral column: on homeotic transformations, pathologies and prenatal selection. Evol Biol. 2012;39(4):456-471. Available in: https://doi.org/10.1007/s11692-0129196-1

7. Torres Cueco R. La columna cervical: síndromes clínicos y su tratamiento manipulativo. México, D.F.: Médica Panamericana; 2006. p. 518.

8. Sharma DK, Vishnudutt, Sharma V, Rathore M. Prevalence of 'Cervical Rib' and its association with gender, body side, handedness and other thoracic bony anomalies in a population of Central India. Indian Journal of Basic and Applied Medical Research. 2014;3(2):593-597.

9. Urschel HC Jr, Razzuk MA. Neurovascular compression in the thoracic outlet: changing management over 50 years. Ann Surg.
1998;228(4):609-617. Available in: https://doi.org/10.1097/00000658199810000-00017

10. Kim TI, Sarac TP, Orion KC. Intravascular ultrasound in venous thoracic outlet syndrome. Ann Vasc Surg. 2019;54:118-122. Available in: https://doi.org/10.1016/j.avsg.2018.08.077

11. Leonhard V, Smith R, Caldwell G, Smith HF. Anatomical variations in the brachial plexus roots: implications for diagnosis of neurogenic thoracic outlet syndrome. Ann Anat. 2016;206:21-26. Available in: https://doi.org/10.1016/j.aanat.2016.03.011

12. Pellerin M, Kimball Z, Tubbs RS, Nguyen S, Matusz P, Cohen-Gadol $A A$, et al. The prefixed and postfixed brachial plexus: a review with surgical implications. Surg Radiol Anat. 2010;32(3): 251-260. Available in: https://doi.org/10.1007/s00276-009-0619-3

13. Levine NA, Rigby BR. Thoracic outlet syndrome: biomechanical and exercise considerations. Healthcare (Basel). 2018;6(2):68. Available in: https://doi.org/10.3390/healthcare6020068

14. Morel J, Pirvu A, Elie A, Gallet N, Magne JL, Spear R. Functional results of cervical rib resection for thoracic outlet syndrome: impact on professional activity. Ann Vasc Surg. 2019;56:233-239. Available in: https://doi.org/10.1016/j.avsg.2018.09.007

15. Umerani MS, Ashqan Y, Burhan H, Darwish AAQ, Mohammed HR, Alhaji Z. Excision of cervical rib through supraclavicular approach. J Rare Disord Diagn Ther. 2017;3(2):10. Available in: https://doi. org/10.21767/2380-7245.100163

16. Nuutinen $\mathrm{H}$, Riekkinen T, Aittola V, Makinen K, Karkkainen JM. Thoracoscopic versus transaxillary approach to first rib resection in thoracic outlet syndrome. Ann Thorac Surg. 2018;105(3):937-942. Available in: https://doi.org/10.1016/j.athoracsur.2017.10.004

17. Maqbool T, Novak CB, Jackson T, Baltzer HL. Thirty-day outcomes following surgical decompression of thoracic outlet syndrome. Hand (N Y). 2019;14(1):107-113. Available in: https://doi. org/10.1177/1558944718798834

18. Jubbal KT, Zavlin D, Harris JD, Liberman SR, Echo A. Morbidity of first rib resection in the surgical repair of thoracic outlet syndrome. Hand (N Y). 2019;14(5):636-640. Available in: https://doi. org/10.1177/1558944718760037

Conflicto de intereses: Los autores declaran no tener conflicto de intereses. 